\title{
Detection of bacterial and intestinal parasitic infections among children under 5 years of age with acute gastroenteritis in a tertiary care hospital in NCR region
}

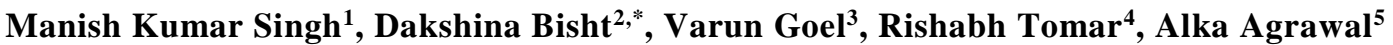 \\ ${ }^{1}$ Ph D Scholar, ${ }^{2,5}$ Professor and Head, ${ }^{3}$ Assistant Professor, ${ }^{4}$ PG Student, ${ }^{1-4}$ Dept. of Microbiology, ${ }^{5}$ Dept. of Paediatrics, \\ Santosh Medical College \& Hospital (Santosh Deemed to be University, NCR Delhi ) Ghaziabad, Uttar Pradesh, India
}

\section{*Corresponding Author:}

Email: dakshinabisht@gmail.com

\begin{abstract}
Introduction: Acute gastroenteritis (AGE) accounts for millions of deaths each year in children, mostly in developing countries. Wide range of pathogens including parasites, bacteria and viruses are responsible for AGE. The aim of this study was to isolate the bacterial and intestinal parasites associated with AGE in children below 5 years of age.

Materials and Methods: A cross sectional study was carried out in Department of Microbiology \& Paediatrics Santosh Medical College and Hospital. 625 children with AGE were enrolled in the study. Stool samples were examined for the presence of parasites and cultured for microbial flora. Drug susceptibility testing for aerobic isolates was performed using Kirby-Bauer disc diffusion method according to CLSI guidelines.

Results: Among the bacterial isolates maximum isolates were Escherichia coli 291 (46.56 \%), of which 32 (5.12\%) samples were identified as Enterohaemorrhagic E.coli (EHEC), 48 (7.68 \%) isolates were identified as Shigella spp. while parasites included Entamoeba histolytica 21 (3.36 \%), followed by Giardia lamblia 14 (2.24 \%), 81.78 E.coli isolates were sensitive to Ampicillin. All the Salmonella spp. isolates were sensitive to Co-trimoxazole.

Conclusion: Most frequently bacterial and parasitic isolates were E.coli Salmonella spp. and Entamoeba histolytica. This gives pre requisite for planning and evaluating interventional programmes associated with diarrhoeal illness and their antimicrobial sensitivity pattern for effective treatment and to decrease disease burden.
\end{abstract}

Keywords: Acute Gastroenteritis (AGE), Diarrhoea, Antimicrobial resistance, Intestinal parasites.

\section{Introduction}

The aetiology of AGE is variable with two main entities: infectious and non-infectious, with the former being the most common. In children acute diarrhoea may have a non-enteric origin, including a range of other infections such as urinary tract infection, pneumonia, otitis media and bacterial sepsis. Noninfectious diarrhoea can result from the intake of toxic food, chemicals, lactose, gluten intolerance or malignancy.

Globally two billion cases of diarrhoeal disease occur every year. ${ }^{1}$ Diarrhoeal disease in developing countries continues to be a big public health problem, where they are estimated to be responsible for 2.5 million infant death per year. ${ }^{2}$ In India one third of total paediatric admission in hospital are due to diarrhoeal disease. ${ }^{3}$ It is also the cause of death in almost $23 \%$ of Indian children who die before the age of five years. ${ }^{4}$

Diarrhoeagenic Escherichia coli represents a leading cause of paediatric diarrhoea while Shigella spp. causes 164.7 million cases of diarrhoea of which 163.2 million occur in developing countries with 1.1 million death each year worldwide mostly in developing countries. ${ }^{5}$ Among the other bacteria Campylobacter jejunii, Salmonella spp. and Vibrio cholerae are also known to cause gastroenteritis. Among parasitic species of particular importance are Entamoeba histolytica, Giardia lamblia, Cryptosporidium parvum and Strongyloides stercoralis.
Among viral pathogens Rotavirus, Norovirus, Astrovirus, Sapovirus, Adenovirus have been associated with AGE.

Most reliable way to diagnose these infections is by detection and identification of the infecting agent. Laboratory diagnosis includes a basic step in the evaluation of disease process at times confirming a presumptive diagnosis or providing evidence of an unsuspected agent of disease. However failure to demonstrate or recover a parasite does not exclude the possibility of infection. Many of these parasites, especially the protozoa can be identified only by microscopic examination. Treatment of infections depends on the infective agent and antimicrobial therapy is not required in some cases as it increases further complications. ${ }^{6}$ The objective of this study was to investigate the microbiological profile of AGE and also analyse the susceptibility pattern of aerobic bacterial isolates.

\section{Materials and Methods}

A cross sectional study was carried out from November 2013 to December 2016 in the Department of Microbiology \& Paediatrics, Santosh Medical College and Hospital. Ethical clearance was obtained from the Institutional Ethical Committee. As per the inclusion criteria the children included with age group less than 5 years with acute gastroenteritis/diarrhoea considered as passage of loose watery stools or an 
increased frequency of stools. As per the exclusion criteria children with diarrhoea more than 14 days, or diarrhoea developing after hospitalization due to any other causes were not included in the present study. After taking written informed consent from parents / guardian at enrolment, stool samples from 625 symptomatic paediatric patients were collected in sterile wide mouth containers (Hi-Media ${ }^{\circledR}$ Mumbai) and examined macroscopically. Microscopic examination for pus cells, charcot-leyden crystals, RBC's ova, trophozoites, cysts and eggs in saline and iodine mount was done. Modified kinyouns's acid fast staining was performed for coccidian parasites. $^{7}$ Concentration technique was used in cases parasites were not seen in saline and iodine mount preparations. Stool motility was performed by hanging drop method and faecal specimens were subjected to culture in order to look for common enteropathogens.

Fresh stool samples were directly cultured on Alkaline Peptone Water (APW), Selenite F broth and Mac Conkey agar and incubated at $37^{\circ} \mathrm{C}$ for $18-24$ hours. After 4 hours of incubation APW was further sub-cultured on Thiosulphate Citrate Bile Salt agar (TCBS) for isolation of Vibrio cholera. After 6 hours of incubation Selenite $\mathrm{F}$ broth was further sub-cultured on Deoxycolate Citrate Agar (DCA) for Salmonella and Shigella spp. Further bacterial isolates were identified by colony characteristics, Gram staining motility and using standard bacteriological procedure.

Antimicrobial susceptibility testing for aerobic bacterial isolates was done by Kirby-Bauer disc diffusion method following Clinical Laboratory Standards Institute (CLSI) guidelines. Antibiotic used were Ciprofloxacin (5 $\mu \mathrm{g} / \mathrm{disc})$, Gentamycin (10 $\mu \mathrm{g} / \mathrm{disc})$, Nalidixic acid $(30 \mu \mathrm{g} / \mathrm{disc})$, Cefotaxime (30 $\mu \mathrm{g} / \mathrm{disc}$ ), Ampicillin (10 mcg/disc), Co-trimoxazole (30 $\mu \mathrm{g} / \mathrm{disc})$, Norfloxacin $(25 \mu \mathrm{g} / \mathrm{disc})$.The data was analysed by using Statistical Package for Social Sciences (SPSS) version 11 and the prevalence of organisms was determined and expressed in percentage.

\section{Results}

Of the 625 children in the age group of 0 to 5 years having AGE, male children were 360 (57.6 \%) while female children were $265(42.4 \%)$. Of the total cases enrolled 370 (59.2 \%) children belonged to rural background while 255 (40.8\%) belonged to urban background (Fig. 1). Most of the cases seen were between age groups 7 to 12 months of age (Table-1).

Of the 625 samples, $291(46.56 \%)$ were positive for Escherichia coli of which $32(5.12 \%)$ were identified as Enterohaemorrhagic E.coli (EHEC) 48 $(7.68 \%)$ as Shigella spp. $7(1.12 \%)$ as Salmonella spp. $16(2.56 \%)$ as Klebsiella spp. $4(0.64)$ as Proteus spp. while only one $(0.6 \%)$ sample was positive for Vibrio cholera. $43(6.88 \%)$ samples were positive for different parasites which included Entamoeba histolytica 21 (3.36 \%), Giardia lamblia 14 (2.24\%) Ascaris lumbricoides $03(0.48 \%)$ Hymenolepis nana 03 (0.48 $\%)$ (Fig. $2 \& 3$ ) and two (0.32\%) samples were positive for Cryptosporidium parvum.

Among the bacterial isolates E.coli were sensitive to Ampicillin (81.78 \%), Ciprofloxacin (36.42 \%), Nalidixic acid $(21.30 \%)$, Co-trimoxazole $(61.51 \%)$, Cefotaxime $(31.95 \%)$ and Gentamycin $(67.69 \%)$ (Fig. 4). All Shigella spp. isolates were sensitive to Ciprofloxacin and Gentamycin but resistant to Cotrimoxazole and Ampicillin (Fig. 5). All the Salmonella spp. isolates were sensitive to Ampicillin, Cefotaxime and Ciprofloxacin but resistant to Co-trimoxazole (Fig. $6)$.

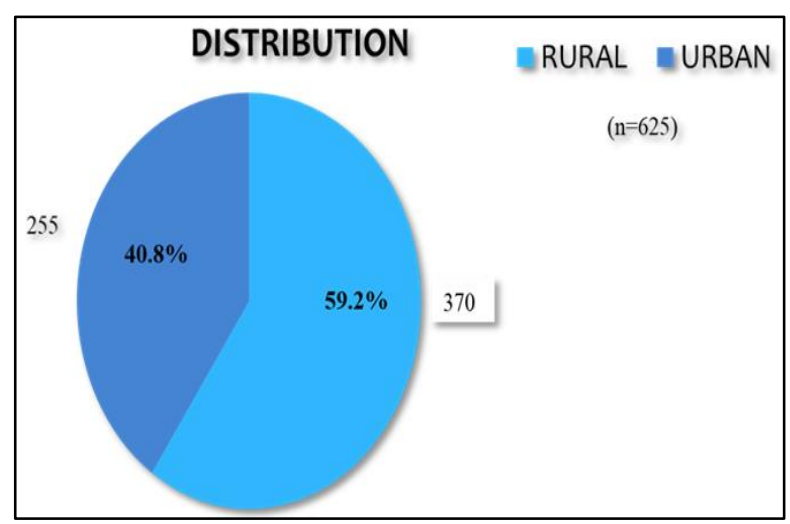

Fig. 1: Demographic distribution of AGE Cases

Table 1: Age and Sex wise distribution of cases

\begin{tabular}{|c|c|c|c|c|}
\hline \multirow{2}{*}{$\begin{array}{l}\text { Age in } \\
\text { Months }\end{array}$} & \multicolumn{2}{|c|}{ Cases } & \multicolumn{2}{c|}{ Sex } \\
\cline { 2 - 5 } & No. & \% & Male (\%) & $\begin{array}{l}\text { Female } \\
(\mathbf{\%})\end{array}$ \\
\hline $0-6$ & 103 & 16.48 & $71(11.36)$ & $32(5.12)$ \\
\hline $7-12$ & 342 & 54.72 & $190(30.4)$ & $155(24.8)$ \\
\hline $13-24$ & 137 & 21.92 & $73(11.68)$ & $61(9.76)$ \\
\hline $25-60$ & 043 & 06.88 & $26(4.16)$ & $17(2.72)$ \\
\hline Total & 625 & & $360(57.6)$ & $265(42.4)$ \\
\hline
\end{tabular}

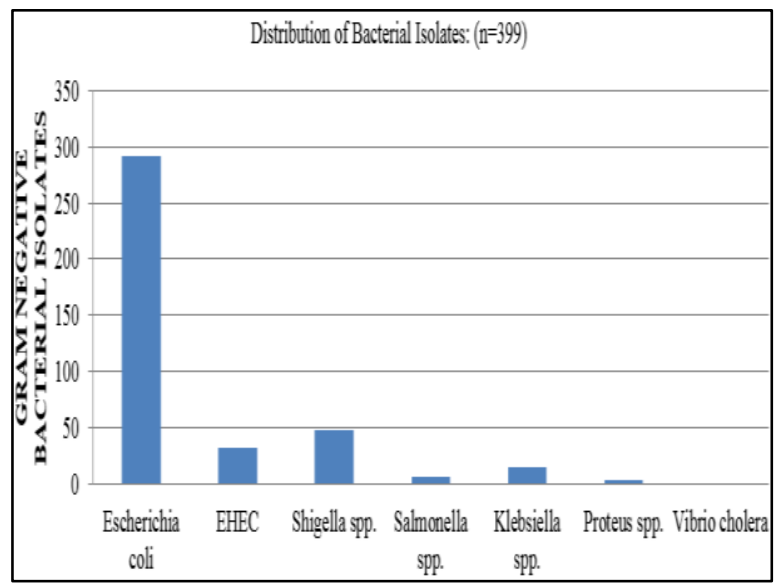

Fig. 2: Distribution of Bacterial Isolates $(n=399)$ 


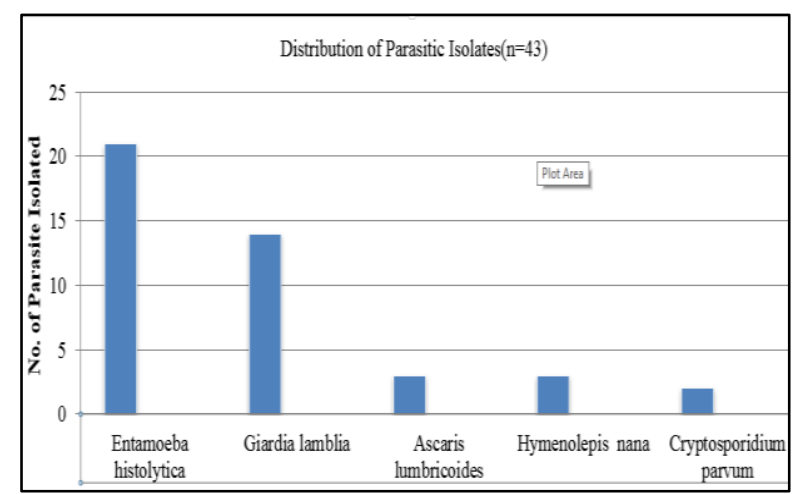

Fig. 3: Distribution of Parasitic Isolates $(n=43)$

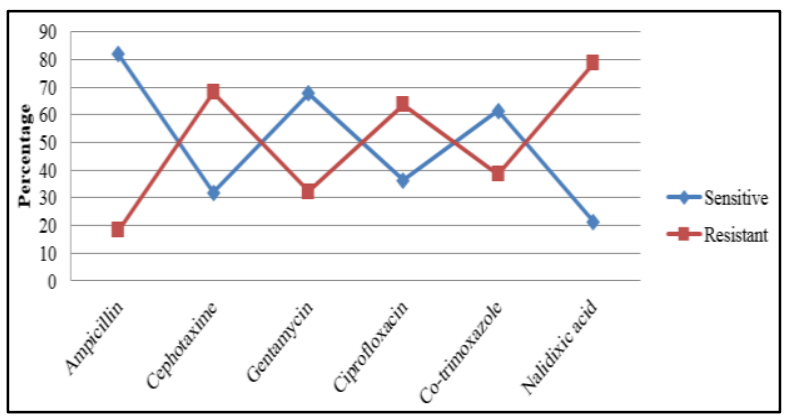

Fig. :4 Sensitivity Patten of E.coli Isolates $(n=291)$

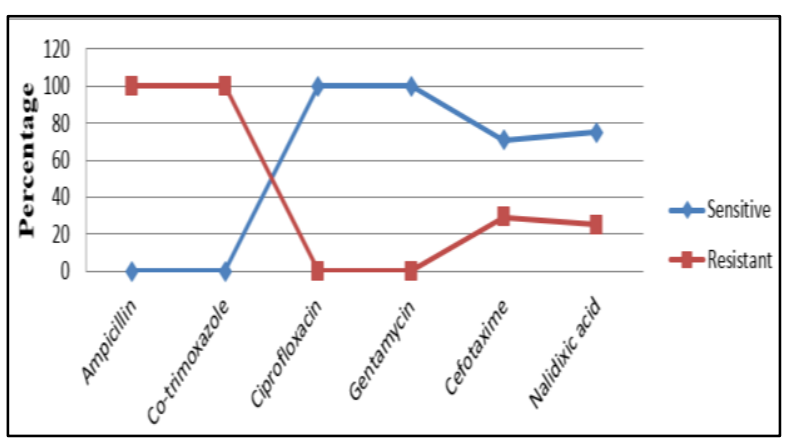

Fig. : 5 Sensitivity Pattern of Shigella spp. Isolates $(n=48)$

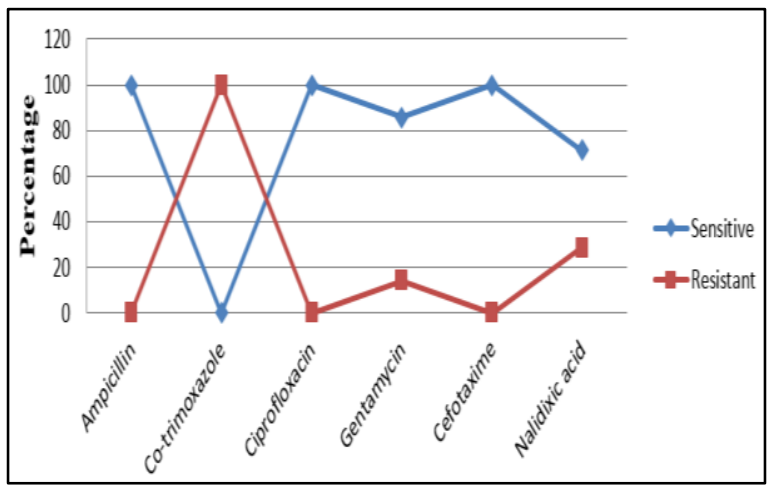

Fig. : 6 Sensitivity Patten of Salmonella spp. Isolates $(\mathbf{n}=7)$

\section{Discussion}

A total of 625 AGE cases were undertaken in the study and it was observed that male children were more affected compared to female children. Most of the children were between the age of 7 to 12 months; which is concordant with the study conducted by Youssef $\mathrm{M}$ et al. ${ }^{8}$ In children there are no clear or validated criteria for antibiotic therapy. Among the bacterial pathogens E.coli were $291(46.56 \%)$ of which $32(5.12 \%)$ were EHEC. Further serotyping of E.coli was not done due to limited availability of antisera. EHEC were higher in our study than a study conducted by Patrick et al. ${ }^{9}$ This is due to increase in the number of sample size. Detection of EHEC would avoid unnecessary diagnostic imaging and surgeries on patients infected with EHEC. Also it avoids deleterious antimicrobial treatment because quinolones which are prescribed for majority of patients are potent inducers of shiga toxin encoding bacteriophages and toxin production in EHEC. Early detection helps in identification and monitoring of patients at risk of progression to haemolytic uraemic syndrome. Shigella and Salmonella spp. were $7.68 \%$ and $1.12 \%$ which correlates with the study conducted by Albert $\mathrm{J}$ et al ${ }^{10}$ and Nair GB et al. ${ }^{11}$ and Okeke et al. ${ }^{12}$ Klebsiella spp. was $2.56 \%$, Proteus spp. were $0.64 \%$ and Vibrio Cholerae were $0.6 \%$ which is congruent with the study conducted by Ajjampur SSR et al. ${ }^{13}$ for Vibrio cholera.

Among parasites Entamoeba histolytica were 3.36 $\%$ where as in another study conducted by Das $\mathrm{S}$ et al. ${ }^{14}$ $4.8 \%$ was reported however the study group involved adults also. Giardia lamblia were $(2.24 \%)$ which was more than the study conducted by Ajjampur SSR et al. ${ }^{13}$ Ascaris lumbricoides and Hymenolepis nana were 0.48 $\%$ and $0.48 \%$ respectively. $2(0.32 \%)$ were positive for Cryptosporidium parvum. Screening for cryptosporidium should be contemplated in settings of prolonged diarrhoea. Cryptosporidium had significant impact on childhood growth in both symptomatic and asymptomatic infections with greater severity in symptomatic infection than asymptomatic infection. Thus it makes essential to examine for this in immunedeficient children, as well as in immunocompetent children.The infection though more common and prolonged in immune-deficient children, as well as documented in immunocompetent children. Majority of parasitic infections was associated with poverty conditions such as reduce access to safe drinking water, adequate sanitation, hygiene housing and inadequate access to health facilities.

During the past decades resistance to currently used antimicrobial agents among enteric pathogens has increased Salmanzdeh-A et al. ${ }^{15}$ In the present study $81.78 \%$ E.coli isolates were sensitive to Ampicillin $36.42 \%$ to Ciprofloxacin and $61.51 \%$ were sensitive to Co-trimoxazole. However a study conducted by Sudershan et al. ${ }^{16}$ shows that more than $70 \%$ of isolates 
were resistant to Ampicillin, Co-trimoxazole and Cefotaxime.

All Shigella isolates were sensitive to Ciprofloxacin, Gentamycin which correlates with the study conducted by Das S. ${ }^{14}$ and Shapiro Rl et al. ${ }^{17}$ All Salmonella isolates were found to be sensitive to Ciprofloxacin, Cefotaxime, Ampicillin which also correlate with the study conducted by Moyo et al. ${ }^{[18]}$ but in our study all salmonella isolates were also $100 \%$ resistant to Co-trimoxazole. The high rate of resistance to these antibiotic shows may be because of their indiscriminate use.

\section{Conclusion}

Decision to treat a child with AGE and choice of antimicrobial drugs is challenging. Furthermore, infection with multiple pathogens which are common among children with diarrhoea therefore complicates the treatment and the antimicrobial resistance pattern should also be considered in antibiotic choice. Improvement in the sanitation and hygiene is also essential in decreasing the morbidity due to bacterial and parasitic pathogens. High rate of resistance of bacterial isolates to Ampicillin and Co-trimoxazole was seen which recommends continuous antibiotic surveillance. Further study on the prevalence of other pathotype Escherichia coli is required. We believe that the data of this study may contribute to an effective management of AGE.

\section{References}

1. WHO: Global networks for surveillance of rota virus gastroenteritis, 2001- 2008. Weekly Epidemiological Report. 2008;83(47):421-28.

2. Kosek M, Bern C, Guerrant RL. The global burden of diarrhoeal disease, as estimated from studies published between 1992 and 2000. Bull World Health Organisation. 2003;81(3):197-204.

3. Park K. Acute Diarrhoeal Diseases, in: Park K, editor. Parks textbook of preventive and social medicine, $24^{\text {th }}$ ed. Jabalpur. Banarasidas Bhanot. 2017:236-48.

4. Jones G, Schultink, W. \& Babille, M. Child survival in India. Indian J Pediatr. 2006;73(6):479-87.

5. Kotloff KL, Nataro JP, Blackwelder WC, Nasrin D, Farag $\mathrm{TH}$, Panchalingam S, et al. Burden and aetiology of diarrhoeal disease in infants and young children in developing countries (the Global Enteric Multicenter Study, GEMS): a prospective, case-control study. Lancet 2013;382(9888):209-22.

6. Kaili fan, Arthur J. Morris, L. Barth Reller. Application of Rejection Criteria for Stool Cultures for Bacterial Enteric Pathogens, J Clin Microbiol. 1993;31(8):2233-35.

7. Lynne Shore Garcia, Diagnostic medical parasitology, 5th edition, ASM Publication.

8. Mohammad Youssef, Abdallah Shurman, MarieElisabeth Bougnoux, Mohammad Rawashdeh, Stephane Bretagne, Nancy Strockbine, Bacterial, viral and parasitic enteric pathogens associated with acute diarrhoea in hospitalized children from northern Jordan; FEMS Immunol Med Microbiol. 2000;28:257-63.

9. Patrick J, Gavin, Lance R. Peterson, Anna C. Pasquariello, Joanna Blackburn, Mark G. Humming, Kuo J. Kuo. et al. Evaluation of performance and potential clinical impact of prospect Shiga Toxin Escherichia coli Microplate Assay for Detection of shiga toxin producing E.coli in stool samples. J Clin Microbiol. 2004;42(4):1652-56.

10. John Albert, A.S.G Faruque, S.M. Faruque et al. Case control study of Enteropathogens associated with childhood diarrhoea in Dhaka, Bangladesh. J Clin Microbiol. 1999;37:3458-64.

11. Gopinath Balakrish Nair, Thandavarayan Ramamurthy, Mihir Kumar Bhattacharya, Triveni Krishnan, Sandipan Ganguly, Dhira Rani Saha, et. al. Emerging trends in the etiology of enteric pathogens as evidenced from an active surveillance of hospitalized diarrhoeal patients in Kolkata, India; Gut Pathogens. 2010;2:4:1-13.

12. Iruka N Okeke, Adebayo Lamikandra, Harmut Steinruck, James B. Kaper. Characterization of E.coli strains from cases of childhood diarrhea in provincial southwestern Nigeria. J Clin Microbiol. 2000;38(1):7-12.

13. S. S. R. Ajjampur, P. Rajendran, S. Ramani, I. Banerjee, B. Monica, P. Sankaran, et. al. Closing the diarrhoea diagnostic gap in Indian children by the application of molecular techniques. J Med Microbiol. 2008;57:136488.

14. S. Das, R. Saha, S. Singhal. Enteric pathogens in North Indian patients with diarrhoea; Ind J comm med. 2007;32(1):27-31.

15. Salmanzdeh-Ahrabi, S., Jafari, F., Habibi, E., Irajian, G.R., Aslani, M.M. and Baghbani-Arani, F. Serotype distribution and antimicrobial resistance rates of Shigella spp. isolates in Tehran, Iran. Mikrobiyol. Bul 2007; 41:453-57.

16. 16.Sudershan RV, Kumar RN, Kulkarni Bharathi, Kashinath L, Bhaskar V, Polasa K. E. coli pathotypes and their antibiotic resistance in young children with diarrhea in Hyderabad, India. Int J Curr Microbiol Appl Sci 2014;3(9):647-54.

17. Roger L. Shapiro, Lata Kumar, Penny Phillips-Howard, Joy G. Wells, Penny Adcock, John Brooks, et. al. Antimicrobial resistant bacterial diarrhoea in rural western Kenya. J Infect dis. 2001;183(11);1701-4.

18. Sabrina J. Moyo, Njolstad Gro, Mecky I. Matee, Jesse Kitundu, Helge Myrmel, Haima Mylvaganam, et. al. Age specific aetiological agents of diarrhoea in hospitalized children aged less than five years in Dar es Salaam. Tanzania BMC Paediatrics. 2011;11(19):1-6. 